\title{
7. Literatur
}

Adolf-Grimme-Institut (2002): Ein Jahr nach dem Terroranschlag vom 11. September: 10 Thesen des Adolf-Grimme-Instituts zum gegenwärtigen Fernsehjournalismus. Marl: Adolf-GrimmeInstitut.

Adorján, Johanna (2006): Meine Zeit ist jetzt zu Ende. Vergewaltigte bei Kerner, zwanzig Kochshows, überall dieselben Nasen. Alfred Biolek über das Fernsehen von heute. Frankfurter Allgemeine Sonntagszeitung, Nr. 38, 24. September, S. 33.

AGF (2009a): Marktanteile der AGF- und Lizenzsender im Tagesdurchschnitt 2008. OnlineVeröffentlichung der Arbeitsgemeinschaft der Arbeitsgemeinschaft Fernsehforschung (AGF). Erhältlich: www.agf.de/daten/zuschauermarkt/marktanteile.

AGF (2009b): Entwicklung der durchschnittlichen Sehdauer pro Tag/Person in Minuten. OnlineVeröffentlichung der Arbeitsgemeinschaft der Arbeitsgemeinschaft Fernsehforschung (AGF). Erhältlich: www.agf.de/daten/zuschauermarkt/sehdauer.

Albrecht, Hans-Jörg (2004): Öffentliche Meinung, Kriminalpolitik und Kriminaljustiz. In: Michael Walter, Harald Kania und Hans-Jörg Albrecht: Alltagsvorstellungen von Kriminalität. Individuelle und gesellschaftliche Bedeutung von Kriminalitätsbildern für die Lebensgestaltung (Kölner Schriften zur Kriminologie und Kriminalpolitik, Band 5). Münster: Lit, S. 491-520.

Altheide, David L. (2002): Creating Fear: News and the Construction of Crisis. New York: Walter de Gruyter.

Altheide, David L. und andere (2001): News Constructions of Fear and Victim: An Exploration Through Triangulated Qualitative Document Analysis. Qualitative Inquiry, 7. Jg. (3), S. 304321.

Althoff, Martina (1999): Die Wirklichkeit der Medien und die Berichterstattung über Kriminalität. Eine Bestandsaufnahme. Leviathan. Zeitschrift für Sozialwissenschaft, 27. Jg. (4), S. 479-500.

ARD (2008): Mediennutzung und Freizeitbeschäftigung 2007. ARD-Online-Veröffentlichung. Erhältlich:

www.ard.de/intern/basisdaten/mediennutzung/mediennutzung_20und_20freizeitbesch_26_2322 8_3Bfti/-/id=54992/15w2mhl/index.html.

ARD (2009): Durchschnittliche Nutzungsdauer von Fernsehen, Hörfunk und Internet in Minuten/Tag. ARD-Online-Veröffentlichung. Erhältlich:

www.ard.de/intern/basisdaten/onlinenutzung/onlinenutzung_3A_20zeiten_20und_20dauer//id=55190/1198aso/index.html

Bartel, Ralph (1997): Fernsehnachrichten im Wettbewerb. Die Strategien der öffentlich-rechtlichen und privaten Anbieter. Köln, Weimar, Wien: Böhlau.

Barton, Allen H. und Paul F. Lazarsfeld (1979): Einige Funktionen von qualitativer Analyse in der Sozialforschung. In: Christel Hopf und Elmar Weingarten (Hrsg.): Qualitative Sozialforschung, Stuttgart: Klett-Cotta, S. 41-89.

Batson, C. Daniel (1991): The altruism question: Toward a socialpsychological answer. Hillsdale (New Jersey, USA): Lawrence Erlbaum.

Batson, C. Daniel und Tecia Moran (1999): Empathy-induced altruism in a prisoner's dilemma. European Journal of Social Psychology, 29. Jg. (7), S. 909-924.

Bauer, Raymond A. (1964): The Obstinate Audience. The Influence Process from the Point of View of Social Communication, American Psychologist, 19. Jg. (5), S. 319-328 
Baumann, Ulrich (1995): Das Bild des Opfers in der Kriminalitätsdarstellung der Medien. Ergebnisse einer Untersuchung. Mainzer Schriften zur Situation von Kriminalitätsopfern, Band 13. Mainz: Weißer Ring.

Baurmann, Michael C. (2004): Monster und Supermänner? Mythen und Realitäten über Tatverdächtige, Straftäter und die polizeiliche Ermittlungsarbeit. In: Michael Walter, Harald Kania und Hans-Jörg Albrecht (Hrsg.): Alltagsvorstellungen von Kriminalität. Individuelle und gesellschaftliche Bedeutung von Kriminalitätsbildern für die Lebensgestaltung (Kölner Schriften zur Kriminologie und Kriminalpolitik, Band 5). Münster: Lit, S. 435-455.

Bayerisches Landeskriminalamt (1995): Ausländer als Opfer von Straftaten. München: Bayerisches Landeskriminalamt.

Bentele, Günter (1993): Wie wirklich ist die Medienwirklichkeit? Einige Anmerkungen zum Konstruktivismus und Realismus in der Kommunikationswissenschaft. In: ders. und Manfred Rühl: Theorien öffentlicher Kommunikation. Problemfelder, Positionen, Perspektiven. München: Ölschläger, S. 152-171.

Bereswill, Mechthild (1999): Gefängnis und Jugendbiographie: Qualitative Zugänge zu Jugend, Männlichkeitsentwürfen und Delinquenz. JuSt-Bericht Nr. 4. KFN Forschungsberichte Nr. 78. Hannover: Kriminologisches Forschungsinstitut Niedersachsen e.V. (KFN).

Bergmann, Jörg R. (1985): Flüchtigkeit und methodische Fixierung sozialer Wirklichkeit. Aufzeichnungen als Daten der interpretativen Soziologie. In: Wolfgang Bonß (Hrsg.): Entzauberte Wissenschaft. Zur Relativität und Geltung soziologischer Forschung. Göttingen: Otto Schwartz, S. 299-320.

Beuler, Roman und Jürgen Wilke (1998): Produktion von Fernsehnachrichten. Eine Untersuchung der Bebilderung der heute-Nachrichtensendung des ZDF. In: Jürgen Wilke (Hrsg.): Nachrichtenproduktion im Mediensystem. Von den Sport- und Bilderdiensten bis zum Internet. Köln: Böhlau, S. 255-277.

Bielstein, Klaus (1996): Polizei und Medien. In: Michael Kniesel, Edwin Kube und Manfred Murck (Hrsg.). Handbuch für Führungskräfte der Polizei: Wissenschaft und Praxis. Lübeck: SchmidtRömhild, S.1035-1058.

Blöbaum, Bernd (1994): Journalismus als soziales System: Geschichte, Ausdifferenzierung und Verselbständigung. Opladen: Westdeutscher Verlag.

Böckelmann, Frank (1993): Journalismus als Beruf. Bilanz der Kommunikatorforschung im deutschsprachigen Raum von 1945 bis 1990. Konstanz: UVK.

Böhm, Andreas (1994): „Grounded Theory - Wie aus Texten Modelle und Theorien gemacht werden“. In: Andreas Böhm, Andreas Mengel und Thomas Muhr (Hrsg.). Texte verstehen. Konzepte, Methoden, Werkzeuge. Konstanz: Universitätsverlag, S. 121-139.

Böhm, Andreas (2004): Theoretisches Kodieren: Textanalyse in der Grounded Theory. In: Uwe Flick, Ernst von Kardorff und Ines Steinke (Hrsg.): Qualitative Forschung. Ein Handbuch. Reinbek bei Hamburg: Rowohlt Taschenbuch, S. 475-484.

Bohn, Irina, Franz Hamburger und Kerstin Rock (1995): Polizei und Presse. Eine Untersuchung zum „staatlich genährten“ Rassismus am Beispiel der Berichterstattung über Sinti und Roma, Jahrbuch für Antisemitismusforschung, 4. Jg., S. 166-183.

Bohnsack, Ralf (2003): Rekonstruktive Sozialforschung: Einführung in qualitative Methoden (5., überarb. Aufl.). Opladen: Leske und Budrich.

Bolz, Norbert (2006): Gute Werte, schlechte Werte. Gesellschaftliche Ethik und die Rolle der Medien. Vortragsmitschrift am 15. September 2006, Tagung der Freiwilligen Selbstkontrolle Fernsehen in Berlin. Erhältlich: www.fsf.de/fsf2/aktivitaeten/bild/tvimpuls/20060915_werte/Vortrag_Bolz_061017.pdf . 
Bonfadelli, Heinz (2004): Medienwirkungsforschung I. Grundlagen und theoretische Perspektiven. 3., überarbeitete Auflage. Konstanz: UVK.

Bortz, Jürgen und Nicola Döring (1995): Forschungsmethoden und Evaluation. 2. Auflage. Berlin, Heidelberg, New York: Springer.

Bourdieu, Pierre (1997): Verstehen. In: Pierre Bourdieu u.a. (Hrsg.): Das Elend der Welt. Zeugnisse und Diagnosen alltäglichen Leidens an der Gesellschaft. Konstanz: UVK, S. 779822.

Bourdieu, Pierre (1998): Über das Fernsehen. Frankfurt am Main: Suhrkamp.

Breed, Warren (1973): Soziale Kontrolle in der Redaktion: Eine funktionale Analyse. In: Jörg Aufermann, Hans Bohrmann und Rolf Sülzer (Hrsg.): Gesellschaftliche Kommunikation und Information. Frankfurt/Main: Athenäum, S. 365-378.

Broeck, Andreas van der (1996): „,Recht brisant“ - Kriminalität im Fernsehen: Beobachtungen und Analysen zu einem Fernsehgerichtsmagazin. Dissertation an der Johannes GutenbergUniversität Mainz. Mainz: Selbstverlag.

Brosius, Hans-Bernd (2001): Stabilität und Wandel: Inhalte und Darstellungsformen von Fernsehnachrichten. In: Frank Marcinkowski (Hrsg.): Die Politik der Massenmedien. Heribert Schatz zum 65. Geburtstag. Köln: Herbert von Halem, S. 115-141.

Brüchert, Oliver (2005): Autoritäres Programm in aufklärerischer Absicht: Wie Journalisten Kriminalität sehen. Münster: Westfälisches Dampfboot.

Bruns, Thomas (1998): Veränderungen der Gewaltberichterstattung im politischen Informationsprogramm des öffentlich-rechtlichen und privaten Fernsehens von 1986-1994. Eine Längsschnittanalyse. Köln: Herbert von Halem.

Bruns, Thomas und Frank Marcinkowski (1997): Politische Information im Fernsehen. Eine Längsschnittstudie zur Veränderung der Politikvermittlung in Nachrichten und politischen Informationssendungen (Schriftenreihe Medienforschung der Landesanstalt für Rundfunk Nordrhein-Westfalen, Band 21). Opladen: Leske und Budrich.

Buckalew, James K. (1969): A Q-Analysis of Television News Editors' Decisions. Journalism Quarterly, 46. Jg. (1), S. 135-137.

Bundeskriminalamt (1989): Polizeiliche Kriminalstatistik (PKS) 1988. Wiesbaden: Bundeskriminalamt.

Bundeskriminalamt (2007): Polizeiliche Kriminalstatistik (PKS) 2006. Wiesbaden: Bundeskriminalamt. Erhältlich: www.bka.de/pks/pks2006/index.html.

Bundeskriminalamt (2008): Polizeiliche Kriminalstatistik (PKS) 2007. Wiesbaden: Bundeskriminalamt. Erhältlich: www.bka.de/pks/pks2007/download/pks-jb_2007_bka.pdf.

Bundesministerium des Innern (Hrsg.) (1996): Medien und Gewalt. Bonn: Selbstverlag.

Bundesministerium des Innern und Bundesministerium der Justiz (Hrsg.) (2006): Zweiter Periodischer Sicherheitsbericht. Erhältlich: www.bka.de/lageberichte/ps/psb2_langfassung.pdf.

Bundesregierung (1998): Bericht der Bundesregierung über die Lage der Medien in der Bundesrepublik Deutschland 1998. Medienbericht 1998. Bundestagsdrucksache 13/10650 vom 18.5.. Berlin: Deutscher Bundestag.

Burkart, Roland (2004): Kommunikationswissenschaft. Grundlagen und Problemfelder. Umrisse einer interdisziplinären Sozialwissenschaft (4., überarb. u. akt. Aufl.). Wien, Köln und Weimar: Böhlau.

Burkhart, Dagmar (2002): Wenn Medien zum Pranger werden. Rede im Rahmen des Symposiums „Wenn (nur) der Ruf zählt: Führung im Zeitalter riskanter Öffentlichkeit, veranstaltet von der Fairness-Stiftung am 7.12.2002 in Frankfurt am Main. Erhältlich:

www.fairness-stiftung.de/FairPreisStatements2002.asp?Statement=BurkhartStatement. 
Campbell, Joseph (2005): Der Heros in tausend Gestalten, 3. Auflage, Frankfurt am Main: Insel

Cantor, Joanne (2003): Media violence and interventions: The roles of communication and emotion. In: Bryant Jennings, David Roskos-Ewoldsen und dies. (Hrsg.): Communication and emotion. Essays in honor of Dolf Zillmann. Mahwah, New Jersey: Erlbaum, S. 287-306.

Carter, Roy E. (1959): Racial Identification Effects Upon the News Story Writer. Journalism Quarterly, 36. Jg. (3), S. 284-290.

Cavender, Gray (2004): Media and Crime Policy. A Reconsideration of David Garland's The Culture of Control. Punishment \& Society, 6. Jg. (3), S. 335-348.

Cavender, Gray und Mark Fishman (Hrsg.) (1998): Entertaining Crime. Television Reality Programs. New York: Aldine du Gruyter.

Cavender, Gray und Lisa Bond-Maupin (1993): Fear and Loathing on Reality Television. An Analysis of „America's Most Wanted” and "Unsolved Mysteries”. Sociological Inquiry, 63. Jg. (3), S. 305-317.

Charlton, Michael (2004): Emotionspsychologische Grundlagen, in: Roland Mangold, Peter Vorderer und Gary Bente: Lehrbuch der Medienpsychologie. Göttingen u.a.: Hogrefe Verlag für Psychologie, S. 129-152.

Chermak, Steven M. (1995): Victims in the News: Crime and the American News Media. Boulder, San Francisco und Oxford: Westview Press.

Chibnall, Steve (1981): The production of knowledge by crime reporters. In: Stanley Cohen und Jock Young (Hrsg.): The manufacture of news: Social problems, deviance and the mass media (2., kompl. überarb. Aufl.). London: Constable, Beverly Hills: Sage, S. 75-97.

Cohen, Stanley (2003): Folk Devils and Moral Panics. 3. Auflage. London: Taylor and Francis.

COMDAT (Communication Data Research) (1993a): Darstellung von Gewalt im Fernsehen. Inhaltsanalyse 11.-17.11.92. Abschlußbericht. Untersuchung im Auftrag von RTL. Münster.

COMDAT (1993b): Darstellung von Gewalt im Fernsehen. Programmanalyse: 20.3 - 3.4.1993. Untersuchung im Auftrag von RTL. 2 Bde. Münster.

Crone, Maren (2005): Packaging Politics: Strategien und Mechanismen medialer Politikvermittlung in deutschen und amerikanischen Nachrichtensendungen. Dissertation an der Wilhelms-Universität zu Münster (Westf.). Münster: Selbstverlag. Erhältlich:

miami.uni-muenster.de/servlets/DerivateServlet/Derivate-2005/packaging_politics.pdf.

Daniel, Matthias (2007): „Die Sender sind nur noch Gefäße“. Im Gespräch mit Hans Mahr. Journalist, 57. Jg. (9), S. 18-21.

Darschin, Wolfgang und Heinz Gerhard (2003): Fernsehgewohnheiten und Fernsehreichweiten im Jahr 2002. Tendenzen im Zuschauerverhalten. media perspektiven, 48. Jg. (4), S. 340-344.

Daschmann, Gregor (2001): Der Einfluss von Fallbeispielen auf Leserurteile: experimentelle Untersuchungen zur Medienwirkung. Konstanz: UVK.

Daschmann, Gregor (2007): Beispielhafte Willkür? Was Journalisten über den Umgang mit Beispielen denken. In: Wiebke Möhring, Walter J. Schütz und Dieter Stürzebecher (Hrsg.): Journalistik und Kommunikationsforschung. Festschrift für Beate Schneider. Berlin: Vistas, S. 169-178.

Daschmann, Gregor und Hans-Bernd Brosius (1997): Ist das Stilmittel die Botschaft? Fallbeispiele in deutschen Fernsehmagazinen. Rundfunk und Fernsehen, 45. Jg. (4), S. 486-504.

Davis, F. James (1952): Crime news in Colorado newspapers. American Journal of Sociology, 57. Jg. (4), S. 325-330.

Davis, Mark H. (1996): Empathy: a social psychological approach. Boulder, Colorado: Westview Press. 
Denzin, Norman K. (1970): The research act: a theoretical introduction to sociological methods. 2. Ausgabe. Chicago: Aldine Publishing Company.

Denzin, Norman K. u. Yvonna S. Lincoln (2000): The handbook of qualitative research (2. Aufl.). Thousand Oaks u.a.: Sage.

Derwein, Christof (1995): Wie wird Kriminalität in der Presse dargestellt, ist die Darstellung wirklichkeitsfremd und gibt es Entsprechungen im Vorstellungsbild der Bevölkerung? Dissertation an der Universität Frankfurt am Main. Frankfurt a. Main: Selbstverlag.

Devereux, Georges (1984): Angst und Methode in den Verhaltenswissenschaften. Übersetzt von Caroline Neubaur und Karin Kersten. Frankfurt am Main: Suhrkamp Taschenbuch.

Diehlmann, Nicole (2003): Journalisten und Fernsehnachrichten. In: Georg Ruhrmann, Jens Woelke, Michaela Maier und dies.: Der Wert von Nachrichten im deutschen Fernsehen. Ein Modell zur Validierung von Nachrichtenfaktoren. (Schriftenreihe Medienforschung der Landesanstalt für Medien Nordrhein-Westfalen, Band 45). Opladen: Leske und Budrich, S. 99-144.

Dixon, Travis L., Daniel Linz (2000): Race and the Misrepresentation of Victimization on Local Television. Communication Research, 27. Jg. (5), S. 547-573.

Dombrowski, Kerstin (2008): Titten, Tiere, Tränen, Tote. Eine Boulevard-Journalistin auf der Jagd. Reinbek bei Hamburg: Rowohlt Taschenbuch.

Donsbach, Wolfgang (1982): Legitimationsprobleme des Journalismus. Freiburg. München: Karl Alber.

Donsbach, Wolfgang (1983): Journalists' Conception of their Audience. Comparative Indicators for the Way British and German Journalists Define Their Relations to the Public. Gazette, 32. Jg. (1), S. 9-36.

Donsbach, Wolfgang (1987): Journalismusforschung in der Bundesrepublik Deutschland: Offene Fragen trotz „Forschungsboom“. In: Jürgen Wilke (Hrsg.): Zwischenbilanz der Journalistenausbildung. Schriftenreihe der Deutschen Gesellschaft für Publizistik- und Kommunikationswissenschaft, Band 14, S. 105-142.

Donsbach, Wolfgang (1993): Journalismus versus journalism - ein Vergleich zum Verhältnis von Medien und Politik in Deutschland und in den USA. In: Wolfgang Donsbach u.a. (Hrsg.): Beziehungsspiele - Medien und Politik in der öffentlichen Diskussion. Fallstudien und Analysen. Gütersloh: Verlag Bertelsmann Stiftung. S. 283-315.

Donsbach, Wolfgang (1999): Journalismus und journalistisches Berufsverständnis. In: Jürgen Wilke (Hrsg.): Mediengeschichte der Bundesrepublik Deutschland. Köln, Weimar, Wien: Böhlau, S. 489-517.

Donsbach, Wolfgang (2002): Psychologische Faktoren hinter Nachrichtenentscheidungen. In: Ute Nawratil, Philomen Schönhagen und Heinz Starkulla jr. (Hrsg.): Medien und Mittler sozialer Kommunikation. Festschrift für Hans Wagner. Leipzig: Leipziger Universitätsverlag, S. 203224.

Döpfner, Mathias (2005, 13.8.): „Wir sind anders“. Spiegel online. Erhältlich: www.spiegel.de/spiegel/0,1518,369624,00.html .

Dörmann, Uwe (2004): Zahlen sprechen nicht für sich. Aufsätze zu Kriminalstatistik, Dunkelfeld und Sicherheitsgefühl aus drei Jahrzehnten. Mit einem Beitrag von Prof. Dr. Wolfgang Heinz. Schriftenreihe Polizei und Forschung, Band 28, herausgegeben vom Bundeskriminalamt, Kriminalistisches Institut. München: Luchterhand.

Dulinski, Ulrike (2003): Sensationsjournalismus in Deutschland. Konstanz: UVK.

Eckert, Thomas und Joachim Huber (2007, 22. August): „Sie müssen mir die Füße küssen“. Im Gespräch mit Roger Schawinski. Tagesspiegel. Erhältlich: www.tagesspiegel.de/mediennews/Sat-1; art15532,2362934. 
Egg, Rudolf (2003): Kriminalität, Kriminologie und die Medien. In: Edwin Kube, Hans Schneider und Jürgen Stock (Hrsg.): Kriminologische Spuren in Hessen. Freundesgabe für Arthur Kreuzer zum 65. Geburtstag. Frankfurt: Verlag für Polizeiwissenschaft, S. 111-122.

Eilders, Christiane (1996): The Role of News Factors in Media Use. Veröffentlichung der Abteilung Öffentlichkeit und soziale Bewegungen des Forschungsschwerpunkts Sozialer Wandel, Institutionen und Vermittlungsprozesse des Wissenschaftszentrums Berlin für Sozialforschung. Erhältlich: bibliothek.wz-berlin.de/pdf/1996/iii96-104.pdf.

Eilders, Christiane (1997): Nachrichtenfaktoren und Rezeption. Eine empirische Analyse zur Auswahl und Verarbeitung politischer Information. Opladen: Westdeutscher Verlag.

Eilders, Christiane (2006): News factors and news decisions. Theoretical and methodological advances in Germany. Communications - The European Journal of Communication Research, 31. Jg. (1), S. 5-24.

van Eimeren, Birgit und Christa-Maria Ridder (2005): Ergebnisse der ARD/ZDF-Langzeitstudie Massenkommunikation: Trends in der Nutzung und Bewertung der Medien 1970 bis 2005. media perspektiven, 36. Jg. (10), S. 490-504.

Eligmann, Barbara (2001): „Immer noch ein Pfund drauflegen“: Barbara Eligmann über Spielregeln des Boulevardjournalismus, über Erfolgsdruck und das Problem ,heiße Ware“. message - internationale Zeitschrift für Journalismus, 3. Jg. (2), S. 46-47.

Elitz, Ernst (1986): Wider die Ware Nervenkitzel: Zum Problem der Gewalt in Informationssendungen. epd medien, 38. Jg. (82), S. 6-9.

Fenton, Frances (1910): The Influence of Newspaper Presentations Upon the Growth of Crime and Other Anti-Social Activity. The American Journal of Sociology, 16. Jg. (3), S. 342-371.

Ferriani, Lino (1897): Entartete Mütter. Eine psychisch-juridische Abhandlung. Aus dem Italienischen von Alfred Ruhemann (Originaltitel: Madri snaturate). Berlin: Cronbach.

Fishman, Mark (1978): Crime Waves as Ideology. Social Problems, 25. Jg. (5), S. 531-543.

Fishman, Mark (1980): Manufacturing the news. Austin (USA): University of Texas Press.

Flegel, Ruth C. und Steven H. Chaffee (1971): Influences of editors, readers, and personal opinions on reporters. Journalism Quarterly, 48. Jg. (4), S. 645-651.

Flick, Uwe (2004): Triangulation: Eine Einführung. Wiesbaden: VS.

Flick, Uwe (2005): Qualitative Sozialforschung: Eine Einführung (vollst. überarb. u. erw. Neuausgabe). Reinbek bei Hamburg: Rowohlt Taschenbuch.

Flick, Uwe (2007): Designing Qualitative Research. London u.a.: Sage.

Friebertshäuser, Barbara (1997): Interviewtechniken - ein Überblick. In: Barbara Friebertshäuser und Annedore Prengel (Hrsg.): Handbuch Qualitative Forschungsmethoden in der Erziehungswissenschaft. Weinheim, München: Juventa, S. 371-395.

Friedrichsen, Gisela (2002): Mord und Medien. In: Rudolf Egg (Hrsg.): Tötungsdelikte - mediale Wahrnehmung, kriminologische Erkenntnisse, juristische Aufarbeitung. Kriminologie und Praxis (Schriftenreihe der Kriminologischen Zentralstelle (Band 36). Wiesbaden: Kriminologische Zentralstelle, S. 21-30.

Friedrichsen, Gisela (2004): „Unheilige Allianzen“ und die Macht der Bilder: Einige Bemerkungen zur zunehmenden Emotionalisierung der Kriminalberichterstattung. In: Michael Walter, Harald Kania und Hans-Jörg Albrecht (Hrsg.): Alltagsvorstellungen von Kriminalität. Individuelle und gesellschaftliche Bedeutung von Kriminalitätsbildern für die Lebensgestaltung (Kölner Schriften zur Kriminologie und Kriminalpolitik, Band 5). Münster: Lit, S. 199-207. 
Friedrichsen, Mike und Stefan Jenzowsky (1995): Methoden und Methodologie. Ein Vergleich ausgewählter Studien der 90er Jahre zur Gewalt in den Medien. In: Mike Friedrichsen und Gerhard Vowe (Hrsg.): Gewaltdarstellung in den Medien. Theorien, Fakten, Analysen. Opladen: Westdeutscher Verlag, S. 292-332.

Fröhlich, Romy (1998): Tatort Nachbarschaft: Frauen und Männer als Opfer und Täter in der lokalen Gewaltberichterstattung. Publizistik, 43. Jg. (4), S. 376-394.

Früh, Werner (1991): Medienwirkungen. Das dynamisch-transaktionale Modell. Theorie und empirische Forschung. Opladen: Westdeutscher Verlag.

Früh, Werner (1994): Realitätsvermittlung durch Massenmedien. Die permanente Transformation der Wirklichkeit. Opladen: Westdeutscher Verlag.

Früh, Werner (2001): Gewaltpotentiale des Fernsehangebots. Programmangebot und zielgruppenspezifische Interpretation. Wiesbaden: Westdeutscher Verlag.

Früh, Werner (2007): Inhaltsanalyse. Theorie und Praxis. Konstanz: UVK.

Früh, Werner und Klaus Schönbach (1982): Der dynamisch-transaktionale Ansatz. Ein neues Paradigma der Medienwirkungen. Publizistik, 27. Jg. (1), S. 74-88.

Früh, Werner und Klaus Schönbach (2005): Der dynamisch-transaktionale Ansatz III: Eine Zwischenbilanz. Publizistik, 50. Jg. (1), S. 4-20.

Funkkorrespondenz (2008): Fernsehjahr 2007: ARD bleibt Marktführer vor ZDF. Funkkorrespondenz, 56. Jg. (2-3), S. 15-17

Galanis, Georgios N. (1987): Migrantenkriminalität in der Presse. Eine inhaltsanalytische Untersuchung dargestellt am Beispiel der Zeitschriften Stern und Quick von 1960-1982. Berlin: Express-Edition.

Galtung, Johan und Mari Holmboe Ruge (1965): The Structure of Foreign News. The Presentation of the Congo, Cuba and Cyprus Crisis in Four Norwegian Newspapers. Journal of Peace Research, 2. Jg. (1), S. 64-91.

Gangloff, Tilmann (2007): Alles Quote - oder was? CUT, 11. Jg. (11), S. 20-25.

Garland, David (2002): The Culture of Control: Crime and Social Order in Contemporary Society. Oxford: Oxford University Press.

Garofalo, James (1981): Crime and the Mass Media: A Selective Review of Research. Journal of Research in Crime and Delinquency, 18. Jg. (2), S. 319-348.

Gerbner, George (1970): Cultural Indicators: The Case of Violence in Television Drama. The Annals of the American Academy of Political and Social Science, 69. Jg. (1), S. 69-81.

Gerbner, George und Larry Gross (1976): Living With Television. The Violence Profile. Journal of Communication, 26. Jg. (2), S. 173-199.

Gerbner, George, Larry Gross, Nancy Signorielli und Michael Morgan (1980): Television Violence, Victimization, and Power. American Behavioral Scientist, 23. Jg. (5), S. 705-716.

Gerbner, George, Larry Gross, Michael Morgan und Nancy Signorielli (1994): Growing up with Television: The Cultivation Perspective. In: Jennings Bryant und Dolf Zillmann (Hrsg.): Media Effects. Advances in Theory and Research. Hillsdale, New Jersey, Hove, United Kingdom: Lawrence Erlbaum Associates, Publishers, S. 17-41.

Gerhard, Heinz (1999): Programmanalysen im Vergleich. Anmerkungen zu Unterschieden in Methode, Aufgabenstellung und Ergebnissen. media perspektiven, 44. Jg. (7), S. 340-344.

Gerhard, Heinz (2008): Fernsehen: Leitmedium im digitalen Zeitalter? Kurzvortrag auf dem Symposium des Hans-Bredow-Instituts: Medienrepertoires sozialer Milieus im medialen Wandel - Perspektiven einer übergreifenden Nutzungsforschung, 11. und 12. 9. 2008, Universität Hamburg. Hamburg: Hans-Bredow-Institut. 
GfK Fernsehforschung (2005): Fernsehzuschauerforschung in Deutschland. Tägliche Informationen über das Fernsehpublikum in Deutschland. Nürnberg: GfK Fernsehforschung $\mathrm{GmbH}$. Erhältlich: www.gfk.com/imperia/md/content/gfk_fernsehforschung/fefo-imagebroschuere-d_juni_2005_.pdf

Gieber, Walter (1956): Across the Desk: A Study of 16 Telegraph Editors. Journalism Quarterly, 33. Jg. (4), S. 423-432.

Glaser, Barney G. und Anselm L. Strauss (2005): Grounded theory: Strategien qualitativer Forschung (2., korr. Aufl.) Bern: Huber.

Glasersfeld, Ernst von (2006): Einführung in den radikalen Konstruktivismus. In: Paul Watzlawick (Hrsg.): Die erfundene Wirklichkeit: Wie wissen wir, was wir zu wissen glauben? Beiträge zum Konstruktivismus. 18. Auflage. München: Piper, S. 16-38.

Gläser, Jochen und Grit Laudel (2004): Experteninterviews und qualitative Inhaltsanalyse als Instrumente rekonstruierender Untersuchungen. Wiesbaden: VS.

Gleich, Uli (1995): Das Angebot von Gewaltdarstellungen im Fernsehen. In: Mike Friedrichsen und Gerhard Vowe (Hrsg).: Gewaltdarstellung in den Medien. Theorien, Fakten, Analysen. Opladen: Westdeutscher Verlag, S. 145-165.

Glotz, Peter und Wolfgang R. Langenbucher (1993): Der missachtete Leser. Zur Kritik der deutschen Presse. Mit Vorworten der Autoren zur Neuausgabe 1993 und zur Originalausgabe 1969. München: Reinhard Fischer.

Gniffke, Kai (2007): Mitja. Eintrag im Tagesschau-Blog, 27. Februar 2007, 21:26 Uhr. Erhältlich: blog.tagesschau.de/?p=330.

Graber, Doris Appel (1980): Crime News and the Public. New York: Praeger.

Grimm, Jürgen (1999): Fernsehgewalt. Zuwendungsattraktivität, Erregungsverläufe, sozialer Effekt. Zur Begründung und praktischen Anwendung eines kognitiv-physiologischen Ansatzes der Medienrezeptionsforschung am Beispiel von Gewaltdarstellungen. Opladen/Wiesbaden: Westdeutscher Verlag.

Grimm, Petra, Katja Kirste und Jutta Weiß (2005): Gewalt zwischen Fakten und Fiktionen. Eine Untersuchung von Gewaltdarstellungen im Fernsehen unter besonderer Berücksichtigung ihres Realitäts- bzw. Fiktionalitätsgrades. Berlin: Vistas.

Grochowski, Tom (2002): The 'tabloid effect' in the O.J. Simpson Case: The National Enquirer and the production of crime knowledge. International Journal of Cultural Studies, 5. Jg. (3), S. 336-356.

Groebel, Jo und Uli Gleich (1990): Medien und Gewalt. media perspektiven, 36. Jg. (11), S. 737741.

Groebel, Jo und Uli Gleich (1993): Gewaltprofil des deutschen Fernsehprogramms: eine Analyse des Angebots privater und öfentlich-rechtlicher Sender. Opladen: Leske und Budrich.

Gross, Kimberly (2006): Covering Crime in Washington, D.C.: Examining the Nature of Local Television News Coverage of Crime and its Effect on Emotional Response (Forschungspapier R-28). Cambridge, USA: Joan Shorenstein Center der John. F. Kennedy School of Government, Harvard University. Erhältlich:

www.ksg.harvard.edu/presspol/research_publications/papers/research_papers/R28.pdf.

Gunter, Barrie (1987): Poor reception: misunderstanding and forgetting broadcast news. Hillsdale (New Jersey, USA): Erlbaum.

Hacker, Friedrich (1988): Gewalt in der Welt - Ursachen - Formen. In: Hans Robert Eisenhauer und Heinz-Werner Hübner (Hrsg.): Gewalt in der Welt - Gewalt im Fernsehen. Mainz: von Hase \& Koehler, S. 17-28. 
Hagemann, Otmar (1993): Wohnungseinbrüche und Gewalttaten: Wie bewältigen Opfer ihre Verletzungen? Eine kriminologische Untersuchung über die Auswirkungen von Straftaten. Hamburger Studien zur Kriminologie, Band 15. Pfaffenweiler: Centaurus.

Hagemann, Otmar (2003): Qualitativ-heuristische Methodologie im Lehr-Dialog. In: ders. und Friedrich Krotz (Hrsg.): Suchen und Entdecken. Beiträge zu Ehren von Gerhard Kleining. Berlin: Rhombos, S. 31-62.

Hajok, Daniel (2004): Jugend und Fernsehinformation. Eine explorativ-deskriptive Studie. Dissertation im Fachbereich Erziehungswissenschaft und Psychologie, Freie Universität Berlin. Berlin: Selbstverlag. Erhältlich: www.diss.fu-berlin.de/2004/126/index.html.

Hakansson Eklund, Jakob (2006): Empathy and viewing the other as a subject. Scandinavian Journal of Psychology, 47. Jg. (5), S. 399-409.

Haller, Michael (2004): Die zwei Kulturen: Journalismustheorie und journalistische Praxis. In: Martin Löffelholz (Hrsg.): Theorien des Journalismus. Ein diskursives Handbuch (2., vollst. überarb. u. erw. Aufl.). Wiesbaden: VS, S. 129-150.

Haller, Michael (2007): Kann gesellschaftliche Kommunikation gelingen? Vom Berichterstatter zum Kommunikator - Überlegungen zu einem überfälligen Funktions- und Rollenwandel im Journalismus. In: Werner Wirth, Hans-Jörg Stiehler und Carsten Wünsch (Hrsg.): Dynamischtransaktional denken. Theorie und Empirie der Kommunikationswissenschaft. Köln: Herbert von Halem, S. 227-251.

Heise, Elke (2000): Sind Frauen mitgemeint? Eine empirische Untersuchung zum Verständnis des generischen Maskulinums und seiner Alternativen. Sprache \& Kognition, 19. Jg. (1-2), S. 3-13.

Herres, Volker (1997): Journalismus, Ethik, Verantwortung, Medien-Zukunft. Vortrag auf dem 2. Hamburger Journalistenforum, veranstaltet von der Fachgruppe Journalismus/dju in Hamburg und der Henri-Nannen-Journalistenschule am 06.09.1997.

Erhältlich: 194.245.102.185/publikationen/m/1997/11/06.html.

Hestermann, Thomas (1997): Verbrechensopfer - Leben nach der Tat. Reinbek bei Hamburg: Rowohlt Taschenbuch.

Hestermann, Thomas (2003): Raub, Gewalt und Nervenkitzel. Jugendgewalt und Jugendkriminalität: Ursachen und Auswege, 10. Auflage. Hilden: Deutsche Polizeiliteratur.

Hestermann (2008): Fernsehbilder des Schreckens und was die Verantwortlichen leitet: Methodentriangulation und computergestützte Datenanalyse, in: Kuckartz, Udo; Thorsten Dresing, Stefan Rädiker und Claus Stefer (Hrsg.) CAQD 2008: 10. MAXQDA-Anwendertagung zur computerunterstützten Analyse qualitativer Daten, Tagungsband. Marburg: Universität Marburg, S. 61-72.

Hestermann, Thomas (2009a): Schatten der Gewalt: Kommerzielle sexuelle Ausbeutung von Minderjährigen in Deutschland, in: UNICEF Deutschland (Hrsg.): UNICEF-Report 2009: Stoppt sexuelle Ausbeutung! Frankfurt am Main: Fischer, S. 61-68.

Hestermann, Thomas (2009b): Das ideale Opfer: jung, weiblich, deutsch. Wie das Fernsehen Gewaltkriminalität darstellt und warum es kaum über Menschenhandel berichtet. Politische Studien, 60. Jg. (427), S. 47-53.

Hickethier, Knut (2002): Das Erzählen der Welt in den Fernsehnachrichten: Überlegungen zu einer Narrationstheorie der Nachrichten. In: Irene Neverla, Elke Grittmann, Monika Pater (Hrsg.): Grundlagentexte zur Journalistik. Konstanz: UVK, S. 657-681. Erhältlich: www.mediacultureonlne.de/fileadmin/bibliothek/hickethier_fernsehnachrichten/hickethier_fernsehnachrichten.html

Höbermann, Frauke (1989): Der Gerichtsbericht in der Lokalzeitung: Theorie und Praxis. BadenBaden: Nomos. 
Hoffmann, Alexander von (1992): Attraktion ohne Recherche. Die Kriminalität als Stoff der Medien. Medium, 22. Jg. (2), S. 58-61.

Hohlfeld, Ralf (2002a): Forschung als Diskussionsanreiz. Optimierung von Sendungen beim „DeutschlandRadio“. Interview mit Ernst Elitz. In: ders., Klaus Meier und Christoph Neuberger (Hrsg.): Innovationen im Journalismus. Forschung für die Praxis. Münster: Lit, S. 211-222.

Hohlfeld, Ralf (2002b): Journalismus für das Publikum? Zur Bedeutung angewandter Medienforschung für die Praxis. In: ders., Klaus Meier und Christoph Neuberger (Hrsg.): Innovationen im Journalismus. Forschung für die Praxis. Münster: Lit, S. 155-202.

Hohlfeld, Ralf (2003): Journalismus und Medienforschung: Theorie, Empirie, Transfer. Konstanz: UVK.

Hohlfeld, Ralf (2005): „Der missachtete Leser revisited“. Zum Wandel von Publikumsbild und Publikumsorientierung im Journalismus. In: Markus Behmer, Bernd Blöbaum, Armin Scholl und Rudolf Stöber (Hrsg.): Journalismus und Wandel: Analysedimensionen, Konzepte, Fallstudien. Wiesbaden: VS, S. 195-224.

Hopf, Christel (1978): Die Pseudo-Exploration - Überlegungen zur Technik qualitativer Interviews in der Sozialforschung. Zeitschrift für Soziologie, 7. Jg. (2), S. 97-115.

Jaeggi, Eva, Angelika Faas und Katja Mruck (1998): Denkverbote gibt es nicht! Vorschlag zur interpretativen Auswertung kommunikativ gewonnener Daten (Veröffentlichungsreihe der Technischen Universität Berlin: Forschungsbericht aus der Abteilung Psychologie im Institut für Sozialwissenschaften, Forschungsbericht Nr. 2-98).

Erhältlich: www.gp.tu-berlin.de/psy7/pub/reports.htm.

Jäger, Siegfried, Ina Ruth, Margret Jäger und Gabriele Cleve (1998): Von deutschen Einzeltätern und ausländischen Banden: eine Diskursanalyse. Jungle World, 2. Jg. (46).

Erhältlich: www.nadir.org/nadir/periodika/jungle_world/_98/46/15b.htm.

Juzas, Stefan (2002): ... und immer an den Leser denken! Die Produktion von Herrschaftswissen durch Nachvollzugsgehilfen. In: Ralf Hohlfeld, Klaus Meier und Christoph Neuberger (Hrsg.): Innovationen im Journalismus. Forschung für die Praxis. Münster: Lit, S. 207-209.

Kaiser, Günther (2002): Medienkriminalität - Spiegel der Wirklichkeit oder Instrument der Kriminalpolitik? Zeitschrift für Rechtspolitik, 35. Jg. (1), S. 30-34.

Kammann, Uwe (2002): Wachschlaf der Ungeheuer. Erfurt, die Medien und gesellschaftliche Moral. epd medien, 50.Jg. (31), S. 3-7. Erhältlich: www.epd.de/medien_index_2279.html.

Kania, Harald (2000): Kriminalitätsdarstellung in den Massenmedien. In: Bundesministerium der Justiz (Hrsg.): Kriminalität in den Medien. Mönchengladbach. Mönchengladbach: ForumVerlag Godesberg, S. 78-97.

Kapuscinski, Ryszard (1999): Die große Reporterarmee: Wie die Medien die Welt beschreiben. Frankfurter Allgemeine Zeitung, 51. Jg. (37), 13. Februar, S. III.

Karl, Claudia (2006): Kriminalitätsberichterstattung im Fernsehen: Eine vergleichende empirische Untersuchung zu den Entscheidungsprozessen und zur Vorgehensweise von Journalisten bei der Kriminalitätsberichterstattung anhand zweier ausgewählter TV-Regionalmagazine in den USA und in Deutschland. Unveröffentlichte Diplomarbeit an der Hochschule Magdeburg-Stendal (FH).

Karstens, Eric und Jörg Schütte (2005): Praxishandbuch Fernsehen. Wie TV-Sender arbeiten. Wiesbaden: VS.

Katz, Jack (1987): What Makes Crime News? Media, Culture and Society, 9. Jg. (1), S. 47-75.

Kelle, Udo (2001): Sociological Explanations between Micro and Macro and the Integration of Qualitative and Quantitative Methods. Forum Qualitative Sozialforschung, 2. Jg. (1). Erhältlich: www.qualitative-research.net/fqs-texte/1-01/1-01kelle-e.htm. 
Kelle, Udo (2007): Die Integration qualitativer und quantitativer Methoden in der empirischen Sozialforschung.Theoretische Grundlagen und methodologische Konzepte. Wiesbaden: VS.

Keppler, Angela (2006): Mediale Gegenwart. Eine Theorie des Fernsehens am Beispiel der Darstellung von Gewalt. Frankfurt am Main: Suhrkamp.

Kepplinger, Hans Mathias in Zusammenarbeit mit Hans-Bernd Brosius, Joachim Friedrich Staab und Günter Linke (1989): Instrumentelle Aktualisierung. Grundlage einer Theorie publizistischer Konflikte. In: Max Kaase und Winfried Schulz (Hrsg.): Massenkommunikation. Theorien, Methoden, Befunde. Sonderheft der Kölner Zeitschrift für Soziologie und Sozialpsychologie, Nr. 30, S. 199-220.

Kepplinger, Hans Mathias (1990): Realität, Realitätsdarstellung und Medienwirkung. In: Jürgen Wilke (Hrsg.): Fortschritte der Publizistikwissenschaft. Freiburg im Breisgau: Alber, S. 39-55.

Kepplinger, Hans Mathias (1992): Ereignismanagement. Wirklichkeit und Massenmedien. Zürich: Edition Interfrom.

Kepplinger, Hans Mathias (1996): Publizistikwissenschaftliche Aspekte der Meinungsvielfalt. In: Hans-Bernd Brosius, Andreas Fahr und Camille Zubayr (Hrsg.): Meinung, Meinungsvielfalt, Meinungsrelevanz. Interdisziplinäre Perspektiven. (Angewandte Medienforschung, Schriftenreihe des Medieninstituts Ludwigshafen, Band 2). München: Reinhard Fischer. S. 2543.

Kepplinger, Hans Mathias (1998a): Der Nachrichtenwert der Nachrichtenfaktoren. In: Christina Holtz-Bacha, Helmut Scherer und Norbert Waldmann (Hrsg.): Wie die Menschen die Welt erschaffen und wie die Menschen darin leben. Opladen: Westdeutscher Verlag 1998, S. 19-38.

Kepplinger, Hans Mathias (1998b): Die Demontage der Politik in der Informationsgesellschaft. Freiburg im Breisgau: Alber, S. 178-205.

Kepplinger, Hans Mathias (2000): Die Entwicklung der Kriminalitätsberichterstattung. In: Bundesjustizministerium (Hrsg.): Kriminalität in den Medien. Mönchengladbach: Forum-Verlag Godesberg, S. 58-77.

Kepplinger, Hans Mathias (2002): Öffentliche Meinung und Gewalt. In: Wilhelm Heitmeyer und John Hagan (Hrsg.): Internationales Handbuch der Gewaltforschung. Opladen: Westdeutscher Verlag, S. 1423-1440.

Kepplinger, Hans Mathias (2004): Problemdimensionen des Journalismus. Wechselwirkung von Theorie und Empirie. In: Martin Löffelholz (Hrsg.): Theorien des Journalismus: Ein diskursives Handbuch. 2. Auflage, Wiesbaden: VS, S. 87-106.

Kepplinger, Hans Mathias (2006): Forschungslogik der Nachrichtenwertforschung. Theoretische Grundlagen. In: Werner Wirth, Andreas Fahr und Edmund Lauf (Hrsg.): Forschungslogik und design in der Kommunikationswissenschaft, Band 2: Anwendungsfelder in der Kommunikationswissenschaft. Köln: Herbert von Halem, S. 15-34.

Kepplinger, Hans-Mathias und Helga Weissbecker (1991): Negativität als Nachrichtenideologie. Publizistik, 36. Jg. (3), S. 330-342.

Kerbel, Matthew Robert (2000): If it bleeds, it leads. An anatomy of television news. Boulder, Colorado (USA): Westview Press.

Kleine, Rolf und Jörg Quoos (2001): Herr Bundeskanzler, geht der Regierung die Puste aus? Interview mit Gerhard Schröder. Bild am Sonntag, 48. Jg. (27), S. 8-9.

Kleining, Gerhard (1982): Umriss zu einer Methodologie qualitativer Sozialforschung. Kölner Zeitschrift für Soziologie und Sozialpsychologie, 34. Jg. (2): S. 224-253. Erhältlich: www.heureka-hamburg.de/UmrissaufsatzKleining.pdf.

Kleining, Gerhard (1991): Methodologie und Geschichte qualitativer Sozialforschung. In: Uwe Flick, Ernst von Kardoff, Heiner Keupp, Lutz von Rosenstiel und Stephan Wolff (Hrsg.): 
Handbuch qualitative Sozialforschung: Grundlagen, Konzepte, Methoden und Anwendungen. München: Beltz Psychologie Verlags Union, S. 11-22.

Erhältlich: www.ssoar.info/ssoar/GetDocument/?resid=3722.

Kleining, Gerhard (1994): Qualitativ-heuristische Sozialforschung. Schriften zur Theorie und Praxis. Hamburg: Rolf Fechner.

Erhältlich: www.ssoar.info/ssoar/GetDocument?resid=773.

Kleining, Gerhard (1995): Lehrbuch Entdeckende Sozialforschung. Von der Hermeneutik zur qualitativen Heuristik (Band 1). Weinheim: Psychologie Verlags Union.

Kleining, Gerhard (1999): Qualitative Sozialforschung. Teil II: Der Forschungsprozess. Hagen: Fernuniversität, Gesamthochschule in Hagen.

Kleining, Gerhard (2001): Offenheit als Kennzeichen entdeckender Forschung. Kontrapunkt: Jahrbuch für kritische Sozialwissenschaft und Philosophie, S. 27-36.

Erhältlich: www.ssoar.info/ssoar/GetDocument?resid=851.

Kleining, Gerhard (2007): Der qualitative Forschungsprozess. In: Gabriele Naderer und Eva Balzer (Hrsg.): Qualitative Marktforschung in Theorie und Praxis. Grundlagen, Methoden und Anwendungen. Wiesbaden: Gabler, S. 189-230.

Erhältlich: www.ssoar.info/ssoar/GetDocument?resid=893.

Kleining, Gerhard und Harald Witt (2000): The Qualitative Heuristic Approach: A Methodology for Discovery in Psychology and the Social Sciences. Rediscovering the Method of Introspection as an Example. Forum Qualitative Sozialforschung / Forum: Qualitative Social Research, 1. Jg. (1). Erhältlich: www.qualitative-research.net/fqs-texte/1-00/1-00kleiningwitte.htm.

Klimmt, Christoph, Verena Pompetzki und Christopher Blake (2008): Geschlechterrepräsentation in Nachrichtentexten. Der Einfluss von geschlechterbezogenen Sprachformen und Fallbeispielen auf den gedanklichen Einbezug von Frauen und die Bewertung der Beitragsqualität. Medien \& Kommunikationswissenschaft, 56. Jg. (1), S. 3-20.

Knipphals, Dirk und Christian Schlüter (1996): „Wahrheit ist nicht zentral". Woher wissen wir das, was wir wissen? Aus den Medien. Und was folgt daraus? Fragen an den Bielefelder Sozialwissenschaftler Niklas Luhmann. Deutsches Allgemeines Sonntagsblatt, 49. Jg. (42).

Köcher, Renate (1985): Spürhund und Missionar. Eine vergleichende Untersuchung über Berufsethik und Aufgabenverständnis britischer und deutscher Journalisten. Dissertation an der Universität München. München: Selbstverlag.

Koelbl, Herlinde (2001): Die Meute. München: von dem Knesebeck.

Krotz, Friedrich (2005): Neue Theorien entwickeln. Eine Einführung in die Grounded Theory, die Heuristische Sozialforschung und die Ethnografie anhand von Beispielen aus der Kommunikationsforschung. Köln: Herbert von Halem.

Krüger, Udo Michael (1994): Gewalt in Informationssendungen und Reality-TV. Quantitative und qualitative Unterschiede im öffentlich-rechtlichen und privaten Fernsehen. media perspektiven, 39. Jg. (2), S. 72-85.

Krüger, Udo Michael (1995): Gewalt in Informationssendungen und Reality-TV. media perspektiven Materialien, 1. Jg. (1).

Krüger, Udo Michael (2000): Unterschiedliches Informationsverständnis im öffentlich-rechtlichen und privaten Fernsehen. media perspektiven, 45. Jg. (7), S. 278-296.

Krüger, Udo Michael (2005): Sparten, Sendungsformen und Inhalte im deutschen Fernsehangebot. media perspektiven, 50. Jg. (5), S. 190-203. 
Krüger, Udo Michael (2006): Jahresbilanz 2005 des InfoMonitors. Fernsehnachrichten bei ARD, ZDF, RTL und SAT.1: Strukturen, Themen und Akteure. media perspektiven, 51. Jg. (2), S. 5074.

Krüger, Udo Michael (2007): InfoMonitor 2006. Fernsehnachrichten bei ARD, ZDF, RTL und SAT.1. media perspektiven, 52. Jg. (2), S. 58-82.

Krüger, Udo Michael (2008): InfoMonitor 2007: Unterschiedliche Nachrichtenkonzepte bei ARD, ZDF, RTL und SAT.1. media perspektiven, 53. Jg. (2), S. 58-83.

Krüger, Udo Michael (2009): InfoMonitor 2008: Fernsehnachrichten bei ARD, ZDF, RTL und Sat.1. media perspektiven, 54. Jg. (2), S. 73-94.

Krüger, Udo Michael (2010): InfoMonitor 2009: Fernsehnachrichten bei ARD, ZDF, RTL und Sat.1. media perspektiven, 55. Jg. (2), S. 50-72.

Krüger, Udo Michael und Thomas Zapf-Schramm (2001): Die Boulevardisierungskluft im deutschen Fernsehen. media perspektiven, 46. Jg. (7), S. 326-344.

Krüger, Udo Michael und Thomas Zapf-Schramm (2003): Inhalte und Gestaltung öffentlichrechtlicher und privater Informationsangebote im Fernsehen. media perspektiven, 48. Jg. (12), S. 534-548.

Krüger, Udo Michael und Thomas Zapf-Schramm (2006): Programmanalyse 2005 von ARD/Das Erste, ZDF, RTL, SAT.1 und ProSieben. Sparten, Sendungsformen und Inhalte im deutschen Fernsehangebot. media perspektiven, 51. Jg. (4), S. 201-221.

Krüger, Udo Michael und Thomas Zapf-Schramm (2007): Programmanalyse von ARD/Das Erste, ZDF, RTL, SAT.1 und ProSieben: Sparten, Sendungsformen und Inhalte im deutschen Fernsehangebot 2006. media perspektiven, 52. Jg. (4), S. 166-186.

Krüger, Udo Michael und Thomas Zapf-Schramm (2008): Sparten, Sendungsformen und Inhalte im deutschen Fernsehangebot 2007. Programmanalyse von ARD/Das Erste, ZDF, RTL, SAT.1 und ProSieben. media perspektiven 53. Jg. (4), S. 166-189.

Kunczik, Michael (1996): Gewalt und Medien (3., überarb. u. akt. Ausg.). Köln, Weimar, Wien: Böhlau.

Kunczik, Michael und Astrid Zipfel (1998): Wirkungen von Gewaltdarstellungen. In: Walter Klingler, Gunnar Roters und Oliver Zöllner (Hrsg.): Fernsehforschung in Deutschland: Themen - Akteure-Methoden (SWR Schriftenreihe, Medienforschung, Band 1). Baden- Baden: Nomos, S. 561- 577.

Kunczik, Michael und Astrid Zipfel (2001): Publizistik. Köln, Weimar, Wien und Böhlau: UTB.

Kunczik, Michael und Astrid Zipfel (2004): Medien und Gewalt. Befunde der Forschung seit 1998. Projektbericht für das Bundesministerium für Familie, Senioren, Frauen und Jugend. Erhältlich: www.bundespruefstelle.de/bpjm/redaktion/PDF-Anlagen/medien-gewalt-befunde-derforschung-sachbericht-langfassung,property=pdf,bereich=bpjm,rwb=true.pdf.

Lamnek, Siegfried (1990): Kriminalitätsberichterstattung in den Medien als Problem. Monatsschrift für Kriminologie und Strafrechtsreform, 73. Jg. (3), S. 163- 176.

Lamnek, Siegfried (2005): Qualitative Sozialforschung. Lehrbuch. 4., vollständig überarbeitete Auflage. Weinheim, Basel: Beltz Psychologie Verlags Union.

Langer, John (1998): Tabloid television. Popular journalism and the 'other news'. London, New York: Routledge.

Lehnert, Sigrun (2004): Forschungsbericht: „Inhaltsanalyse über Kriminalität im FernsehAngebot" anhand der Programmankündigung in der Tagespresse aus drei Stichwochen der Jahre 1985, 1995 und 2003. Im Auftrag des Kriminologischen Forschungsinstituts Niedersachsen. Hannover: Hochschule für Musik und Theater, Institut für Journalistik und Kommunikationsforschung. Unveröffentlicht. 
Lehr, Gernot (2001): Bildberichterstattung der Medien über Strafverfahren. Neue Zeitschrift für Strafrecht, 21. Jg. (2), S. 63-67.

Lesche, Dieter (2001): Fröhlicher Kannibalismus. TV-News in der Krise? message, 3. Jg. (2). S. 43-47.

Lettau von, Antje und Franz Breuer (2007): Forscher/innen-Reflexivität und qualitative sozialwissenschaftliche Methodik in der Psychologie. Journal für Psychologie, 15. Jg. (2). Erhältlich: www.journal-fuer-psychologie.de/jfp-2-2007-3.html.

Leutheusser-Schnarrenberger, Sabine (2000): Medien als Kriminalpolitiker? In: Bundesministerium der Justiz (Hrsg.): Kriminalität in den Medien. Forum-Verlag Godesberg, S. 182-188.

Lippmann, Walter (1997): Public Opinion. New York: Free Press Paperbacks.

Liska, Allen E. und William Baccaglini (1990): Feeling Safe by Comparison: Crime in the Newspapers. Social Problems, 37. Jg. (3), S. 360-374.

Löhr, Holle Eva (2003): Resozialisierung und Medien. In: Heinz Cornel u.a. (Hrsg.): Handbuch der Resozialisierung (2. Aufl.). Baden-Baden: Nomos, S. 529-561.

Lorenz, Dagmar (2002): Journalismus. Stuttgart und Weimar: Metzler.

Ludes, Peter (1993): Von der Nachricht zur News Show. Fernsehnachrichten aus der Sicht der Macher. München: Fink.

Ludes, Peter (1999): Programmgeschichte des Fernsehens. In: Jürgen Wilke (Hrsg.): Mediengeschichte der Bundesrepublik Deutschland. Köln, Weimar und Wien: Böhlau, S. 255-276.

Ludwig, Hans-Werner und Guido Marc Pruys (1998): Gewaltdarstellungen im Fernsehen. Die öffentliche Debatte und die Produktion. In: Walter Klingler (Hrsg.): Fernsehforschung in Deutschland: Themen - Akteure - Methoden (Südwestfunk Schriftenreihe 1, Teilband 2). Baden-Baden: Nomos, S. 579-596.

Luhmann, Niklas (1996): Die Realität der Massenmedien (2., erw. Aufl.). Opladen: Westdeutscher Verlag.

Mach, Ernst (1905): Erkenntnis und Irrtum. Leipzig: Johann Ambrosius Barth. Erhältlich: www.zeno.org/Philosophie/M/Mach,+Ernst/Erkenntnis+und+Irrtum.

Maier, Michaela (2003): Operationalisierung der Inhaltsanalyse. In: Georg Ruhrmann, Jens Woelke, dies. und Nicole Diehlmann: Der Wert von Nachrichten im deutschen Fernsehen. Ein Modell zur Validierung von Nachrichtenfaktoren. (Schriftenreihe Medienforschung der Landesanstalt für Medien Nordrhein-Westfalen, Band 45). Opladen: Leske und Budrich, S. 5198.

Maier, Michaela, Georg Ruhrmann und Kathrin Klietsch (2006): Der Wert von Nachrichten im deutschen Fernsehen: Ergebnisse einer Inhaltsanalyse 1992 - 2004. Düsseldorf: Landesanstalt für Medien Nordrhein-Westfalen.

Erhältlich: www.lfm-nrw.de/downloads/nachrichtenanalyse_1992-2000.pdf.

Maletzke, Gerhard (1978): Psychologie der Massenkommunikation. Theorie und Systematik. Hamburg: Hans-Bredow-Institut.

Maletzke, Gerhard (1998): Kommunikationswissenschaft im Überblick. Grundlagen, Probleme, Perspektiven. Opladen: Westdeutscher Verlag.

Mast, Claudia u.a. (1999): Programmpolitik zwischen Markt und Moral. Entscheidungsprozesse über Gewalt im Deutschen Fernsehen. Eine explorative Studie. Opladen, Wiesbaden: Westdeutscher Verlag.

Mayr, Gaby (2006): Zwischen Emotionen und Rationalität. Im Strafrecht will die Große Koalition lediglich ergänzen und nachbessern. Hintergrund Politik. [Radio] Deutschlandradio KulturSendung, 14. November 2006. Sendemanuskript.

Erhältlich: www.dradio.de/dlf/sendungen/hintergrundpolitik/563105/. 
McManus, John H. (1994): Market-driven journalism: let the citizen beware? Thousand Oaks, California: Sage.

Meier, Klaus (2002): Ressort, Sparte, Team. Wahrnehmungsstrukturen und Redaktionsorganisation im Zeitungsjournalismus. Konstanz: UVK.

Mergenthaler, Erhard unter Mitarbeit von Marie Mühl (1992): Die Transkription von Gesprächen. Eine Zusammenstellung von Regeln mit einem Beispieltranskript (3. Aufl.). Ulm: Ulmer Textbank. Erhältlich: www.jakob.uzh.ch/docs/Transkript-mergenth.pdf.

Merton, Robert K. und Patricia L. Kendall (1979): Das fokussierte Interview. In: Christel Hopf und Elmar Weingarten (Hrsg.): Qualitative Sozialforschung, Stuttgart: Klett-Cotta, S. 171-204.

Meuser, Michael und Nagel, Ulrike (2005): ExpertInneninterviews - vielfach erprobt, wenig bedacht. Ein Beitrag zur qualitativen Methodendiskussion. In: Alexander Bogner, Beate Littig und Wolfgang Menz (Hrsg.): Das Experteninterview: Theorie, Methode, Anwendung. Wiesbaden: VS, S. 71-93.

Mikos, Lothar (1998): Flanieren auf dem Boulevard zwischen Stars und Mordbuben. Themen und Präsentationsformen in Boulevard-Magazinen. tv diskurs - Verantwortung in audiovisuellen Medien, 2. Jg. (5), S. 64-71.

Mikos, Lothar (2003): Von Kriegen und Verbrechen - Ästhetik der Gewaltdarstellung in Nachrichten und anderen dokumentarischen Formaten. tv diskurs - Verantwortung in audiovisuellen Medien, 7. Jg. (24), S. 10-15.

Möhring, Wiebke und Daniela Schlütz (2003): Die Befragung in der Medien- und Kommunikationswissenschaft. Eine praxisorientierte Einführung. Wiesbaden: Westdeutscher Verlag.

Morris, Norval (1997): Crime, the Media and our Public Discourse. In: James Q. Wilson, et al. (Hrsg.): Perspectives on Crime and Justice. 1996-1997 Lecture Series. Washington: National Institute of Justice, S. 99-121. Erhältlich: www.ncjrs.gov/pdffiles/166609.pdf.

Mruck, Katja unter Mitarbeit von Günter Mey (2000): Qualitative Sozialforschung in Deutschland. Forum Qualitative Sozialforschung / Forum: Qualitative Social Research, 1. Jg. (1). Erhältlich: www.qualitative-research.net/index.php/fqs/article/view/1114/2466.

Muckenhaupt, Manfred (1998): Boulevardisierung in der TV-Nachrichtenberichterstattung. In Werner Holly und Bernd Ulrich Biere (Hrsg.): Medien im Wandel, Opladen: Westdeutscher Verlag, S. 113-134.

Neissl, Julia und Rudi Renger (2002): Auf dem Weg zum „Journalismus light“? Zur Popularisierung des Journalismus in Österreich. In: Archim Baum und Siegfried J. Schmidt (Hrsg.): Fakten und Fiktionen: über den Umgang mit Medienwirklichkeiten. Konstanz: UVK, S. 254-270.

Neuberger, Christoph (1996): Journalismus als Problembearbeitung. Objektivität und Relevanz in der öffentlichen Kommunikation. Konstanz: UVK Medien.

Niedersächsische Landesmedienanstalt (Hrsg.) (1999): Die Tyrannei der öffentlichen Intimität und Tabubrüche im Fernsehen. Boulevardmagazine, Talkshows und Comedy. Dokumentation der Tagung der Niedersächsischen Landesmedienanstalt für Privaten Rundfunk (NLM) im Mai 1998 in Hannover. Berlin: Vistas.

Noack, Paul (2002): Das Schweigen der Redseligen: Ein Versuch über journalistische Biographien. In: Ute Nawratil, Philomen Schönhagen und Heinz Starkulla jr. (Hrsg.): Medien und Mittler sozialer Kommunikation. Festschrift für Hans Wagner. Leipzig: Leipziger Universitätsverlag, S. 255-263.

Norek, Tobias (2005): Medienkriminalität in den Köpfen der Menschen: Wirkungen, Faszination, Bedürfnisse. Ein Beitrag zur Erfassung der "positiven" Seite der subjektiven Kriminalität. Dissertation an der juristischen Fakultät der Universität Köln. Aachen: Shaker. 
Obermöller, Bernd und Mirko Gosch (1995): Kriminalitätsberichterstattung als kriminologisches Problem. Kritische Justiz, 28. Jg. (1), S. 45-59.

Ohler, Josef und Dietz Schwiesau (Hrsg.) (2003): Die Nachricht in Presse, Radio, Fernsehen, Nachrichtenagentur und Internet. Ein Handbuch für Ausbildung und Praxis. München: List.

Oliver, Mary Beth (1994): Portrayals of Crime, Race, and Aggression in "Reality-Based" Police Shows: A Content Analysis. Journal of Broadcasting \& Electronic Media, 37. Jg. (2), S. 179192.

Oliver, Mary Beth (2003): Mood management and selective exposure. In: Bryant Jennings, David Roskos-Ewoldsen und Joanne Cantor (Hrsg.): Communication and emotion. Essays in honor of Dolf Zillmann. Mahwah, New Jersey: Erlbaum, S. 85-106.

Östgaard, Einar (1965): Factors Influencing the Flow of News. Journal of Peace Research, 2. Jg. (1), S. 39-63.

Paoli, Letizia (2004): Verbrechensfurcht und organisierte Kriminalität: "Die Russen-Mafia". In: Michael Walter, Harald Kania und Hans-Jörg Albrecht (Hrsg.): Alltagsvorstellungen von Kriminalität. Individuelle und gesellschaftliche Bedeutung von Kriminalitätsbildern für die Lebensgestaltung (Kölner Schriften zur Kriminologie und Kriminalpolitik, Band 5). Münster: Lit, S. 287-310.

Patterson, Thomas E. (2000): Doing well and doing good. How Soft News and Critical Journalism Are Shrinking the News Audience and Weakening Democracy - And What News Outlets Can Do About It. Joan Shorenstein Center on the Press, Politics and Public Policy, John F. Kennedy School of Government, Harvard University. Cambridge, Massachusetts.

Pfeiffer, Christian (2004): Dämonisierung des Bösen. Frankfurter Allgemeine Zeitung, 56. Jg. (55), 5. März, S. 9.

Pfeiffer, Christian (2005): Weniger Verbrecher, mehr Panikmache. Die Zahl schwerer Straftaten geht zurück. Warum wird das verschwiegen? Ein offener Brief. Die Zeit, 60. Jg. (23), 2. Juni, S. 9.

Pfeiffer, Christian, Matthias Kleimann und Michael Windzio (2004): Die Medien, das Böse und wir. $\mathrm{Zu}$ den Auswirkungen der Mediennutzung auf Kriminalitätswahrnehmung, Strafbedürfnisse und Kriminalpolitik. Monatsschrift für Kriminologie und Strafrechtsreform, 87. Jg. (6), S. 415435.

Pfeiffer, Christian, Michael Windzio und Matthias Kleimann (2005): Media Use and its Impacts on Crime. Perception, Sentencing Attitudes and Crime Policy. European Journal of Criminology, 2. Jg. (3), S. 259-285.

Plake, Klaus (2004): Handbuch Fernsehforschung. Wiesbaden: VS.

Pleitgen, Fritz (2000): Einige grundsätzliche Bemerkungen zur Medienverantwortung. In: Bundesministerium der Justiz (Hrsg.): Kriminalität in den Medien. Forum-Verlag Godesberg, S. 189203.

Proctor, Jon L., Diane M. Badzinski und Michelle Johnson (2002): The Impact of Media on Knowledge and Perceptions of Megan's Law. Criminal Justice Policy Review, 13. Jg. (4), S. 356-379.

Project for Excellence in Journalism (2006): The State of the News Media 2006. An Annual Report on American Journalism. Erhältlich: www.stateofthemedia.org/2006/.

Prokop, Dieter (1998): Warum Einschaltquoten und Hitlisten kein demokratisches Bild der Publikumswünsche ergeben. In: Walter Klingler (Hrsg.): Fernsehforschung in Deutschland: Themen - Akteure - Methoden (Südwestfunk Schriftenreihe 1, Teilband 2). Baden-Baden: Nomos, S. 955-966. 
Pürer, Heinz (1997): Zwischen Tradition und Wandel: Zum Stand der Kommunikatorforschung in Deutschland. In: Hermann Fünfgeld und Claudia Mast (Hrsg.): Massenkommunikation. Ergebnisse und Perspektiven. Opladen: Westdeutscher Verlag, S. 89-123.

Raabe, Johannes (2004): Theoriebildung und empirische Analyse: Überlegungen zu einer hinreichend theorieoffenen, empirischen Journalismusforschung. In: Löffelholz Martin (Hrsg.): Theorien des Journalismus. Ein diskursives Handbuch (2., vollst. überarb. u. erw. Aufl.). Wiesbaden: VS, S. 107-127.

Reijnders, Stijn (2005): The people's detective: true crime in Dutch folklore and popular television. Media, Culture \& Society, 27. Jg. (5), S. 635-651.

Reinemann, Carsten (2003): Medienmacher als Mediennutzer. Kommunikations- und Einflussstrukturen im politischen Journalismus der Gegenwart. Köln: Böhlau.

Renger, Rudi (2000): Geringfügige Nachrichten: Populärer Journalismus zwischen wissenschaftlicher Agnosie und theoretischem Pluralismus. In: Ingrid Paus-Haase, Dorothee Schnatmeyer und Claudia Wegener (Hrsg.): Information, Emotion, Sensation: Wenn im Fernsehen die Grenzen zerfließen (Schriften zur Medienpädagogik, Nr. 30). Bielefeld: Gesellschaft für Medienpädagogik und Kommunikationskultur, S. 12-29.

Reuband, Karl-Heinz (1997): Gesellschaftlicher Wandel, Kriminalität und Kriminalitätsfurcht. In: Konrad-Adenauer-Stiftung (Hrsg.): Kriminalität in Städten und Gemeinden - Herausforderung kommunaler Kriminalpolitik (Schriftenreihe Aktuelle Fragen der Politik, Heft 39). Sankt Augustin: Konrad-Adenauer-Stiftung, S. 7-22.

Reuband, Karl Heinz (2000): Kriminalität als Thema ostdeutscher Massenmedien vor und nach der Wende. Eine Analyse Dresdner Tageszeitungen 1988-1994. Kriminologisches Journal, 32. Jg. (1), S. 43-55.

Ridder, Christa-Maria und Bernhard Engel (2005): Ergebnisse der 9. Welle der ARD/ZDFLangzeitstudie zur Mediennutzung und -bewertung. Massenkommunikation 2005: Images und Funktionen der Massenmedien im Vergleich. media perspektiven, 50. Jg. (9), S. 422-448.

Robinson, Gertrude Joch (1973): Fünfundzwanzig Jahre Gatekeeper-Forschung: Eine kritische Rückschau und Bewertung. In: Jörg Aufermann, Hans Bohrmann, Rolf Sülzer (Hrsg.): Gesellschaftliche Kommunikation und Information, Frankfurt am Main: Athenäum, S. 345-355.

Roithmeier, Karl (1994): Der Polizeireporter. Ein Leitfaden für die journalistische Ausbildung. Konstanz: UVK.

Rosengren, Karl Erik (1970): International News: Intra and Extra Media Data. Acta Sociologica, 13. Jg. (2), S. 96-109.

Roshier, Bob (1981): The selection of crime news by the press. In: Stanley Cohen und Jock Young (Hrsg.): The manufacture of news: Social problems, deviance and the mass media (2., kompl. überarb. Aufl.). London: Constable, Beverly Hills: Sage, S. 40-51.

Rössler, Patrick (2005): Inhaltsanalyse. Köln: UTB.

Rössler, Patrick (2006): „Erst mal sehen, was die anderen machen.“ Vielfalt als Qualitätsmerkmal vs. mediale Koorientierung im journalistischen Alltag. In: Siegfried Weischenberg, Wiebke Loosen und Michael Beuthner (Hrsg.): Medien-Qualitäten. Öffentliche Kommunikation zwischen ökonomischem Kalkül und Sozialverantwortung. Konstanz: UVK, S. 223-244.

Rosten, Leo C. (1937): The Social Composition of Washington Correspondents. Journalism Quarterly, 14. Jg. (2), S. 125-132.

Rückert, Sabine (2003): Kriminalität, Medien und Kriminalpolitik. In: Eric Minthe (Hrsg.): Neues in der Kriminalpolitik. Kriminologie und Praxis (Schriftenreihe der Kriminologischen Zentralstelle, Band 42). Wiesbaden: Kriminologische Zentralstelle, S. 39-47.

Ruhrmann, Georg (2005): Nachrichten zum Fürchten. Message, 7. Jg. (1), S. 94-96. 
Ruhrmann, Georg, Jens Woelke, Michaela Maier und Nicole Diehlmann (2003): Der Wert von Nachrichten im deutschen Fernsehen. Ein Modell zur Validierung von Nachrichtenfaktoren. Opladen: Leske und Budrich.

Ruhrmann, Georg und Roland Göbbel (2007): Veränderung der Nachrichtenfaktoren und Auswirkungen auf die journalistische Praxis in Deutschland. Erhältlich:

www.netzwerkrecherche.de/docs/ruhrmann-goebbel-veraenderung-der-nachrichtenfaktoren.pdf.

Sacco, Vincent F. (1995): Media Constructions of Crime. The Annals of the American Academy of Political and Social Science, 106. Jg. (539), S. 141-159.

Saleth, Stephanie (2004): Jugendkriminalität im Spiegel der Lokalpresse: Eine Gegenüberstellung der Berichterstattung des Schwäbischen Tagblatts und der Statistik der Jugendgerichtshilfe Tübingen im Zeitraum von 1975- 2000. Dissertation an der Eberhard Karls-Universität Tübingen. Tübingen: Selbstverlag.

Saxer, Ulrich und Martina Märki-Koepp (1992): Medien-Gefühlskultur: zielgruppenspezifische Gefühlsdramaturgie als journalistische Produktionsroutine. München: Ölschläger.

Scharf, Wilfried, Hans-Ullrich Mühlenfeld und Ralf Stockmann (1999): Zur Kriminalitätsberichterstattung in der Presse. Publizistik, 44. Jg. (4), S. 445-462.

Schawinski, Roger (2007): Die TV-Falle: Vom Sendungsbewusstsein zum Fernsehgeschäft. Zürich: Kein und Aber.

Scheithauer, Ingrid (2001): Wir sind weder Bote noch Botschafter: CNN-International-Chef Chris Cramer über das Selbstverständnis des weltweiten Nachrichtensenders. Frankfurter Rundschau, 20. September.

Scheuch, Ute K. (1986): Wechselwirkungen zwischen Programm-Machern und Hörern, dargestellt am Beispiel der Hörerpost an die politischen Redaktionen und die Nachrichten-Redaktion des Deutschlandfunks. Krefeld: Marchal und Matzenbacher Wissenschaftsverlag.

Scheufele, Bertram (2005): Sexueller Missbrauch: Mediendarstellung und Medienwirkung. Wiesbaden: VS.

Schnatmeyer, Dorothee (2000): Wie Journalisten versuchen, den Rezipienten zu fesseln. Eine Befragung von Mitarbeitern ausgewählter Boulevardmagazine, in: Ingrid Paus-Haase, Dorothee Schnatmeyer und Claudia Wegener: Information, Emotion, Sensation: Wenn im Fernsehen die Grenzen zerfließen. (Schriften zur Medienpädagogik, Nr. 30). Bielefeld: Gesellschaft für Medienpädagogik und Kommunikationskultur, S. 92-113.

Schneider, Beate, Klaus Schönbach und Dieter Stürzebecher (1993a): Westdeutsche Journalisten im Vergleich: jung, professionell und mit Spaß an der Arbeit. Publizistik, 38. Jg. (1), S. 5-30.

Schneider, Beate, Klaus Schönbach, Dieter Stürzebecher (1993b): Journalisten im vereinigten Deutschland. Strukturen, Arbeitsweisen und Einstellungen im Ost-West-Vergleich. Publizistik, 38. Jg. (3), S. 353-382.

Schneider, Beate, Klaus Schönbach und Dieter Stürzebecher (1994): Oberlehrer oder Missionare? Das Selbstverständnis deutscher Journalisten. In: Friedhelm Neidhardt (Hrsg.): Öffentlichkeit, öffentliche Meinung, soziale Bewegungen. Opladen: Westdeutscher Verlag, S. 139-161.

Schneider, Beate (1998): Mediensystem. In: Otfried Jarren, Ulrich Sarcinelli und Ulrich Saxer (Hrsg.): Politische Kommunikation in der demokratischen Gesellschaft. Ein Handbuch. Opladen und Wiesbaden: Westdeutscher Verlag, S. 422-430.

Schneider, Beate, Anne-Katrin Arnold und Werner Greve (2005a): Paradigmenwechsel in der Kriminalitätsberichterstattung. Qualitative und quantitative Veränderungen von 1971 bis 2001. Vortrag bei der Jahrestagung der Deutschen Gesellschaft für Publizistik- und Kommunikationswissenschaft, 4. - 6. Mai 2005, Hamburg. 
Schneider, Beate, Anne-Katrin Arnold und Werner Greve (2005b): Exponentieller Anstieg. Neue Studie zur Berichterstattung über Sexualmorde an Kindern: mehr Beiträge, weniger Emotionen. Message, 7. Jg. (1), S. 97.

Schneider, Wolf und Paul-Josef Raue (2003): Das neue Handbuch des Journalismus. Vollständig überarbeitete und erweiterte Neuausgabe. Reinbek bei Hamburg: Rowohlt Taschenbuch.

Scholl, Armin (2003): Die Befragung. Sozialwissenschaftliche Methode und kommunikationswissenschaftiche Anwendung. Konstanz: UVK.

Schönbach, Klaus und Werner Früh (1984): Der dynamisch-transaktionale Ansatz II: Konsequenzen. Rundfunk und Fernsehen, 32. Jg. (3), S. 314-329.

Schönbach, Klaus und Siegfried Weischenberg (1984): Inter- und Intra-Transaktionen im Medienwirkungsprozess. Publizistik, 29. Jg. (1), S. 88-89.

Schönhagen, Philomen und Hans-Bernd Brosius (2004): Die Entwicklung der Gewalt- und Kriminalitätsberichterstattung im lokalen Raum. Hat sich die Selektionsschwelle langfristig verschoben? Publizistik, 49. Jg. (3), S. 255-274.

Schulenburg, Caroline (2007): Dying to Entertain. Violence on Prime Time Broadcast TV: 1998 to 2006. Los Angeles, California: Parents Television Council. Erhältlich: www.parentstv.org/ptc/publications/reports/violencestudy/DyingtoEntertain.pdf.

Schultheiss, Britta M. (2001): Boulevardmagazine im Spannungsfeld von Quotendruck und Glaubwürdigkeit im Spiegel der Kommunikatoren und Rezipienten. Eine qualitative Analyse. Dissertationsschrift. Salzburg: Universität Salzburg.

Schulz, Winfried (1976): Die Konstruktion von Realität in den Massenmedien. Analyse der aktuellen Berichterstattung. Freiburg, München: Alber.

Schulz, Winfried (1989): Massenmedien und Realität. Die „ptolemäische“ und die „kopernikanische“ Auffassung. In: Max Kaase und Winfried Schulz (Hrsg.): Massenkommunikation. Theorien, Methoden, Befunde. Sonderheft der Kölner Zeitschrift für Soziologie und Sozialpsychologie, S. 135-149.

Schulz, Winfried (1996): Anschauen und Dafürhalten. Inszenierter Augenschein und öffentliche Meinung. epd Kirche und Rundfunk, 47. Jg. (61), S. 5-10.

Schulz, Winfried (1997): Politische Kommunikation. Theoretische Ansätze und Ergebnisse empirischer Forschung zur Rolle der Massenmedien in der Politik. Opladen: Westdeutscher Verlag.

Seven One Media (2006): Altersstruktur des deutschen Fernsehens. Unterföhring: Seven One Media.

Smaus, Gerlinde (1978): Funktion der Berichterstattung über Kriminalität in den Massenmedien. Kriminologisches Journal, 10. Jg. (3), S. 187-201.

Staab, Joachim Friedrich (1990): Nachrichtenwert-Theorie. Formale Struktur und empirischer Gehalt. Freiburg (Breisgau), München: Alber.

Staab, Joachim Friedrich (1998): Faktoren aktueller Berichterstattung. Die NachrichtenwertTheorie und ihre Anwendung auf das Fernsehen. In: Klaus Kamps und Miriam Meckel (Hrsg.): Fernsehnachrichten. Prozesse, Strukturen, Funktionen. Opladen/Wiesbaden: Westdeutscher Verlag.

Staab, Joachim Friedrich (2002): Entwicklungen der Nachrichtenwert-Theorie: Theoretische Konzepte und empirische Überprüfungen. In: Irene Neverla, Elke Grittmann und Monika Pater (Hrsg.): Grundlagentexte zur Journalistik. Konstanz: UVK, S. 608- 618.

Stahlberg, Dagmar und Sabine Sczesny (2001): Effekte des generischen Maskulinums und alternativer Sprachformen auf den gedanklichen Einbezug von Frauen. Psychologische Rundschau, 52. Jg. (3), S. 131-140. 
Steffens, Lincoln (1931): The autobiography of Lincoln Steffens. New York: Harcourt, Brace.

Stieler, Kaspar (1969): Zeitungs Lust und Nutz. Vollständiger Neudruck der Originalausgabe von 1695, hrsg. von Gert Hagelweide. Bremen: Schünemann.

Stipp, Horst (2009): Zehn aktuelle Medientrends in den USA: Verdrängt Online-Sehen die Fernsehnutzung? media perspektiven, 54. Jg. (5), S. 226-232.

Stolle, Peter und Hartmut Volz (1990): „Der Wurm muss schmecken“: RTL-plus-Programmchef Helmut Thoma über Erfolge und Miseren des Privatfernsehens. Der Spiegel, 44. Jg. (42), S. 162-170.

Strauss, Anselm und Juliet Corbin (1996): Grounded theory. Grundlagen qualitativer Sozialforschung. Weinheim: Beltz, Psychologie Verlags Union.

Strübing, Jörg (2004): Grounded theory. Zur sozialtheoretischen und epistemologischen Fundierung des Verfahrens der empirisch begründeten Theoriebildung. Wiesbaden: VS.

Struve, Günter (1998): Die Darstellung von Kriminalität und Gewalt in den Massenmedien. Initiativen und Perspektiven journalistischer Selbstkritik. In: Bundeszentrale für politische Bildung (Hrsg.): Kriminalität und Sicherheit als Thema der politischen Bildung in Europa. Dokumentation von Plenumsvorträgen des europäischen Expertenseminars der Bundeszentrale für Politische Bildung (Bonn) und des Instituuts voor Publiek en Politiek (Amsterdam) in Cadenabbia (Italien), 29. November - 2. Dezember 1996. Bonn, Amsterdam: Selbstverlag, S. 76-84.

Swanson, Charles E. (1955): What They Read in 130 Daily Newspapers. Journalism Quarterly, 32. Jg. (4): S. 411-421.

Teichmann, Jan Andreas (2002): Der dicke Journalistenbauch. Medienforschung als emanzipatorisches Mittel. In: Ralf Hohlfeld, Klaus Meier und Christoph Neuberger (Hrsg.): Innovationen im Journalismus. Forschung für die Praxis. Münster: Lit, S. 203-206.

Tekolf, Oliver (2005): Schillers Pitaval. Merkwürdige Rechtsfälle als ein Beitrag zur Geschichte der Menschheit, verfasst, bearbeitet und herausgegeben von Friedrich von Schiller 1792, kommentiert von Oliver Tekolf. Frankfurt am Main: Eichborn.

Thomas, William Isaac und Dorothy Swaine Thomas (1928): The Child in America. Behavior Problems and Programs. New York: Alfred A. Knopf.

Tiefel, Sandra (2004): Beratung und Reflexion: Eine qualitative Studie zu professionellem Beratungshandeln in der Moderne. Wiesbaden: VS.

Tyndall, Andrew (2009): Tyndall-Report.Year in Review 2008.

Erhältlich: tyndallreport.com/yearinreview2008/. (Ergänzt durch vom Herausgeber freundlicherweise übermitteltes Datenmaterial für die Jahre 1988 bis 2008.)

Ullrich, Carsten G. (1999): „Deutungsmusteranalyse und diskursives Interview“. Zeitschrift für Soziologie, 28. Jg. (6), S. 429-447.

Unz, Dagmar, Frank Schwab und Peter Winterhoff-Spurk (2001): Gewalt in Fernsehnachrichten: Inhalts- und Formalanalysen im Längsschnittvergleich. Vortrag gehalten auf der 2. Tagung der Fachgruppe Medienpsychologie in Landau, 9.-11. September 2001.

Vasterman, Peter L.M. (2005): Media-Hype: Self-Reinforcing News Waves, Journalistic Standards and the Construction of Social Problems. European Journal of Communication, 20. Jg. (4), S. 508-530.

Vogler, Christopher (2004): Die Odyssee des Drehbuchschreibers. Frankfurt am Main: Zweitausendeins. 
Voß, Peter (2002): Die Macht, die Medien, die Moral und die Gewaltbereitschaft. Und wir alle. In: ders. (Hrsg.): Wem gehört der Rundfunk? Medien und Politik in Zeiten der Globalisierung (SWR Schriftenreihe - Medienpolitik 2). Erhältlich:

www.mediaculture-online.de/Autoren-A-Z.253+M58cfbc1395f.0.html

Voß, Peter (1997): Die Information: Das tägliche Neue. In: Ruth Blaes und Gregor Alexander Heussen (Hrsg.): ABC des Fernsehens. Konstanz: UVK, S. 112-123.

Voß, Peter (1988): Müssen - dürfen - bleibenlassen. Gewalt in Nachrichten und Dokumentationen. In: Hans-Robert Eisenhauer und Heinz-Werner Hübner (Hrsg.): Gewalt in der Welt - Gewalt im Fernsehen. Mainz: von Hase \& Koehler, S. 129-148.

Voß, Peter (2008): Wie objektiv können wir sein? Oder: Was ist eigentlich Nachrichtenkompetenz? epd medien, 60. Jg. (12), S. 8-12.

Walter, Michael (1994): Gedanken zur Bedeutung von Kriminalität in den Medien. In: Peter-Alexis Albrecht (Hrsg.): Festschrift für Horst Schüler-Springorum zum 65. Geburtstag. Köln, Berlin, Bonn, München: Heymann, S. 123-136.

Walter, Michael (1999): Von der Kriminalität in den Medien zu einer bedrohlichen Medienkriminalität und Medienkriminologie? DVJJ-Journal, 10. Jg. (4), S. 348-354.

Walter, Michael (2000): Von der Kriminalität in den Medien zu einer Bedrohung durch Medienkriminalität und Medienkriminologie? In: Bundesministerium der Justiz: Kriminalität in den Medien. 5. Kölner Symposium, S. 10-22.

Warken, Bettina (2006): Bilder - der bestimmende Faktor in der Berichterstattung? Diskussionspapier zum 10. Bundeskongress für Politische Bildung, veranstaltet von der Bundeszentrale für politische Bildung vom 2. - 4. März 2006 in Mainz. Erhältlich: www.bpb.de/files/LQ7Y5C.pdf.

Warren, Carl (1934): Modern News Reporting. Veröffentlicht im Auftrag des United States Armed Forces Institute. New York City: Harper \& Brothers.

Watzlawick, Paul (Hrsg.) (2006): Die erfundene Wirklichkeit: Wie wissen wir, was wir zu wissen glauben? Beiträge zum Konstruktivismus (18., unveränd. Aufl.). München: Piper.

Watzlawick, Paul (2004): Wie wirklich ist die Wirklichkeit? Wahn, Täuschung, Verstehen (30., unveränd. Aufl.). München: Piper.

Weber, Stefan (2000): Was steuert Journalismus? Ein System zwischen Selbstreferenz und Fremdsteuerung. Konstanz: UVK.

Wegener, Claudia (1994): Reality-TV: Fernsehen zwischen Emotion und Information? (Schriften der Gesellschaft für Medien und Kommunikationskultur GMK, Band 7). Opladen: Leske und Budrich.

Weischenberg, Siegfried (1995): Journalistik: Theorie und Praxis aktueller Medienkommunikation. Band 2: Medientechnik, Medienfunktionen, Medienakteure. Opladen und Wiesbaden: Westdeutscher Verlag.

Weischenberg, Siegfried (1998): Journalistik. Theorie und Praxis aktueller Medienkommunikation. Band 1: Mediensysteme, Medienethik, Medieninstitutionen (2., überarb. u. akt. Aufl.). Opladen, Wiesbaden: VS.

Weischenberg, Siegfried (2001): Nachrichten-Journalismus. Anleitungen und Qualitäts-Standards für die Medienpraxis. Unter Mitarbeit von Judith Rakers. Opladen und Wiesbaden: Westdeutscher Verlag.

Weischenberg, Siegfried (2005): Der Schein trügt. Die Zeit, 60. Jg. (41), 6. Oktober.

Weischenberg, Siegfried und Armin Scholl (1989): Kommunikationserwartungen und Medieneffekte: Wie Publikumsvariablen Wirkungsabläufe beeinflussen können. Rundfunk und Fernsehen, 37. Jg. (4), S. 421-434. 
Weischenberg, Siegfried und Armin Scholl (1992): Dispositionen und Relationen im Medienwirkungsprozess. Theoretische Exploration und empirische Evidenz für ein Interprendenzmodell zu den Folgen vermittelter Kommunikation. In: Winfried Schulz (Hrsg.): Medienwirkungen. Einflüsse von Presse, Radio und Fernsehen auf Individuum und Gesellschaft. Weinheim u.a.: VCH, Acta Humaniora, S. 91-107.

Weischenberg, Siegfried und Armin Scholl (1998): Journalismus in der Gesellschaft: Theorie, Methodologie und Empirie. Opladen: Westdeutscher Verlag.

Weischenberg, Siegfried, Susanne von Bassewitz und Armin Scholl (1989): Konstellationen der Aussageentstehung. Zur Handlungs- und Wirkungsrelevanz journalistischer Kommunikationsabsichten. In: Max Kaase und Winfried Schulz (Hrsg.): Massenkommunikation. Theorien, Methoden, Befunde. Sonderheft der Kölner Zeitschrift für Soziologie und Sozialpsychologie, S. 280-300.

Weischenberg, Siegfried, Maja Malik und Armin Scholl (2006a): Die Soffleure der Mediengesellschaft. Report über die Journalisten in Deutschland. Konstanz: UVK.

Weischenberg, Siegfried, Maja Malik und Armin Scholl (2006b): Zentrale Befunde der aktuellen Repräsentativbefragung deutscher Journalisten: Journalismus in Deutschland 2005. media perspektiven, 37. Jg. (7), S. 346-361.

Welch, Kelly (2007): Black Criminal Stereotypes and Racial Profiling. Journal of Contemporary Criminal Justice, 23. Jg. (3), S. 276-288.

Welt (2004): „taff" statt „Tagesschau": Wie Jugendliche fernsehen. Welt online, 30. Juni 2004. Erhältlich:www.welt.de/print-

welt/article324215/Taff_statt_Tagesschau_Wie_Jugendliche_fernsehen.html

Westerstahl, Jorgen und Folke Johansson (1986): News Ideologies as Molders of Domestic News. European Journal of Communication, 1. Jg. (1), S. 133-149.

White, David Manning (1950): The „Gate Keeper“: A Case Study in the Selection of News. Journalism Quarterly, 27. Jg. (3), S. 383-390.

Wiedebusch, Jutta (1989): Selbstverständnis und Rezipientenbilder von Hörfunkjournalisten. Europäische Hochschulschriften (Reihe XL Kommunikationswissenschaft und Publizistik, Band 19), Frankfurt am Main u.a.: Peter Lang.

Wilke, Jürgen (1984): Nachrichtenauswahl und Medienrealität in vier Jahrhunderten. Eine Modellstudie zur Verbindung von historischer und empirischer Publizistikwissenschaft. Berlin, New York: de Gruyter.

Windzio, Michael und Matthias Kleimann (2006): Die kriminelle Gesellschaft als mediale Konstruktion? Mediennutzung, Kriminalitätswahrnehmung und Einstellung zum Strafen. Soziale Welt, 57. Jg. (2), S. 193-215.

Windzio, Michael, Julia Simonson, Christian Pfeiffer und Matthias Kleimann (2007): Kriminalitätswahrnehmung und Punitivität in der Bevölkerung - welche Rolle spielen die Massenmedien? Ergebnisse der Befragungen zu Kriminalitätswahrnehmung und Strafeinstellungen 2004 und 2006. KFN-Forschungsbericht, Nr. 103. Hannover: Kriminologisches Forschungsinstitut Niedersachsen e.V. (KFN).

Winterhoff-Spurk, Peter (1986): Fernsehen. Psychologische Befunde zur Medienwirkung. Bern, Stuttgart, Toronto: Hans Huber.

Winterhoff-Spurk, Peter (1994): Gewalt in Fernsehnachrichten. In: Michael Jäckel und Peter Winterhoff-Spurk (Hrsg.): Politik und Medien. Analysen zur Entwicklung der politischen Kommunikation. Berlin: Vistas, S. 55-70.

Winterhoff-Spurk, Peter, Dagmar Unz und Frank Schwab (2005): Häufiger, schneller, variabler. Ergebnisse einer Längsschnittuntersuchung über Gewalt in TV-Nachrichten. Publizistik, 50. Jg. (2), S. 225-237. 
Witt, Harald (2004): Von der kommerziellen Marktforschung zur akademischen Lehre - eine ungewöhnliche Karriere. Gerhard Kleining im Interview mit Harald Witt. Forum Qualitative Sozialforschung, 5. Jg. (3). Erhältlich: nbn-resolving.de/urn:nbn:de:0114-fqs0403404.

Witzel, Andreas (2000, Januar): Das problemzentrierte Interview. Forum Qualitative Sozialforschung / Forum: Qualitative Social Research, 1. Jg. (1). Erhältlich: www.qualitativeresearch.net/fqs-texte/1-00/1-00witzel-d.htm.

Zachert, Hans-Ludwig (1994): Erfahrungen der Polizei mit den Medien. Eine aktuelle Bestandsaufnahme und die Suche nach besseren Wegen. Kriminalistik, 48. Jg. (11), S. 682-692.

ZDF (2008): Die Darstellung von Migration und Integration in den ZDF-Programmen: Status quo und Perspektiven. Mainz: Zweites Deutsches Fernsehen. Erhältlich:

www.unternehmen.zdf.de/fileadmin/files/Download_Dokumente/DD_Das_ZDF/Migration_und _Integration_im_ZDF-Programm.pdf.

Zenz, Gisela (1978): Einleitung zur deutschen Ausgabe. In: Ray. E. Helfer und C. Henry Kempe: Das geschlagene Kind. Mit einer Einleitung von Gisela Zenz. Frankfurt am Main: Suhrkamp Taschenbuch, S. 17-34.

Zillmann, Dolf (2004): Emotionspsychologische Grundlagen. In: Roland Mangold, Peter Vorderer und Gary Bente: Lehrbuch der Medienpsychologie. Göttingen u.a.: Hogrefe Verlag für Psychologie, S. 101-128.

Zubayr, Camille und Heinz Gerhard (2007): Fernsehgewohnheiten und Fernsehreichweiten im Jahr 2006. Tendenzen im Zuschauerverhalten. media perspektiven, 38. Jg. (4), S. 189-199.

Zubayr, Camille und Stefan Geese (2009): Ergebnisse einer Repräsentativbefragung zur Bewertung der Fernsehnachrichten: Die Informationsqualität der Fernsehnachrichten aus Zuschauersicht. media perspektiven, 40. Jg. (4), S. 158-173. 
